The Journal of Geometric Analysis

Volume 17, Number 2, 2007

\title{
Hyper-Lagrangian Submanifolds of Hyperkähler Manifolds and Mean Curvature Flow
}

\author{
By Naichung Conan Leung and Tom Y. H. Wan
}

\begin{abstract}
In this article, we define a new class of middle dimensional submanifolds of a Hyperkähler manifold which contains the class of complex Lagrangian submanifolds, and show that this larger class is invariant under the mean curvature flow. Along the flow, the complex phase map satisfies the generalized harmonic map heat equation. It is also related to the mean curvature vector via a first order differential equation. Moreover, we proved a result on nonexistence of Type I singularity.
\end{abstract}

A hyperkähler manifold $M$ is a Riemanninan manifold with holonomy $\operatorname{Sp}(n)$. It admits a complex structure $J$ and a holomorphic symplectic form $\Omega_{J} \in \Omega^{2,0}(M)$ because $\operatorname{Sp}(n)=$ $\mathrm{U}(2 n) \cap \mathrm{Sp}(2 n, \mathbb{C})$. In fact it admits a 2-sphere family of such structures, called the twistor family $\mathbb{S}^{2}$. Hyperkähler geometry arises naturally in many moduli problems, see, for example, $[1,7]$ and it is also intimately related to physical theory with $N=4$ supersymmetries, see, for example, [6]. A submanifold $L$ in $M$ is called a complex Lagrangian submanifold if it is Lagrangian with respect to $\Omega_{J}$ for some $J \in \mathbb{S}^{2}$. Since $\Omega_{J}$ is a complex form, Lagrangian respect to $\Omega_{J}$ implies two independent conditions. This is more restrictive than the usual meaning of being Lagrangian in a symplectic manifold. Complex Lagrangian submanifold with respect to $\Omega_{J}$ is always a $J$-complex submanifold of $M$. In particular, it is calibrated by the Kähler form $\omega_{J}$ and an absolute minimal submanifold in $M$. The geometry of such submanifolds are studied in $[8,9]$, for instance.

Since we have a 2-sphere family of holomorphic symplectic structures $\Omega_{J}$ with $J \in \mathbb{S}^{2}$ on $M$, we could relax the assumption to the tangent spaces

$$
T_{x} L \subset T_{x} M
$$

\footnotetext{
Math Subject Classifications. 53C26, 58J35.

Key Words and Phrases. Hyperkähler manifolds, mean curvature flow.

Acknowledgements and Notes. First author was partially supported by Earmarked Grants of Hong Kong CUHK2060275; second author was partially supported by Earmarked Grants of Hong Kong CUHK4291/00P; the authors would like to thank the referee for helpful comments.
}

(1) 2007 The Journal of Geometric Analysis 
to be a complex Lagrangian subspace with respect to $\Omega_{J(x)}$ with varying $J(x) \in \mathbb{S}^{2}$. Such an $L$ is called a hyper-Lagrangian submanifold of $M$ and the above map $x \rightarrow J(x)$ is called the complex phase map

$$
J: L \rightarrow \mathbb{S}^{2}
$$

It is not difficult to see that this notion is defined for a larger class of manifolds, namely Quaternionic-Kähler manifolds, i.e., Riemannian manifolds with holonomy $\operatorname{Sp}(n) \operatorname{Sp}(1)$. For example, $\mathbb{C P}^{n} \subset \mathbb{H} \mathbb{P}^{n}$ and any surface in an oriented four manifold are examples of hyperLagrangian submanifolds.

In this article we study the geometry of hyper-Lagrangian submanifolds in a hyperkähler manifold. We prove the formula relating the mean curvature vector $H$ of $L$ and the complex phase map $J$,

$$
\left.\partial J=\frac{i}{2} H\right\lrcorner \Omega_{J} .
$$

In particular, if $L$ is minimal and the cohomology class $[J] \in H^{2}(L, \mathbb{Z})$ vanishes, then $L$ is a complex Lagrangian submanifold of $M$. This class $[J]$ is called the complex Maslov index of $L$.

We show that the mean curvature flow of a hyper-Lagrangian submanifold $L$ in $M$ preserves the hyper-Lagrangian condition. To prove this, we need to couple the mean curvature flow of $L$ with a harmonic map flow for $J$ and derive appropriate estimates in order to use a maximum principle argument as in [11].

We also show that if the image of $J$ lies in a hemisphere of $\mathbb{S}^{2}$, then this remains so under the mean curvature flow. Furthermore, Type I singularity will not occur in this situation.

When the hyperkähler manifold is of dimension four, or more generally a Kähler Einstein four manifold, these results were obtained by Jingyi Chen [2] and Mutao Wang [13] independently. In this case, the above hemisphere condition is equivalent to the surface being a symplectic submanifold in the Kähler Einstein four manifold.

Another well-behaved class of middle dimensional submanifolds under mean curvature flow are Lagrangian submanifolds in a Calabi-Yau manifold[2, 11, 12, 13]. The results there are completely analogous to those we obtain in this article. This is not a coincidence as our results are the quaternion version of their complex geometric statements. The comparisons of geometry defined over different normed algebras were studied by the first author in [10]. The following table compares the corresponding notions used in this article:

\begin{tabular}{|c|c|}
\hline Complex $\mathbb{C}$ & Quaternion $\mathbb{H}$ \\
$\cap$ Lagrangian Submanifold & Hyper-Lagrangian Submanifold \\
$\cap$ & $\cap$ \\
Kähler Manifold & Quaternionic-Kähler Manifold \\
$\cap$ & $\cap$ \\
Calabi-Yau Manifold & Hyperkähler Manifold \\
\hline
\end{tabular}

Remark that given any hyper-Lagrangian submanifold $L$ in a hyperkähler manifold $M$, suppose that $\operatorname{Re}\left(\Omega_{J(x)}\right)$ is constant for all $x \in L$. Then $L$ is also a Lagrangian submanifold in $M$, 
regarded as a Calabi-Yau manifold via a natural inclusion $\mathrm{Sp}(n) \subset \mathrm{SU}(2 n)$. In this circumstance, our results for hyper-Lagrangian submanifolds in hyperkähler manifolds are indeed the same as the corresponding results for Lagrangian submanifolds in Calabi-Yau manifolds.

Our article will be organized as follows. We will first prove some formulae which are needed in the rest of the article in Section 2. In Section 3, we study the mean curvature flow and show that the hyper-Lagrangian condition is preserved. After this, we study the compatibility condition for a hyper-Lagrangian submanifold and prove the formula (1.1) in Section 4. Finally, we give a brief discussion on the regularity of mean curvature flow of a hyper-Lagrangian submanifold in Section 5.

\section{Terminologies and basic computations}

Let $\left(M^{4 n}, \bar{g}\right)$ be a hyperkähler manifold, $L^{2 n} \subset M$ be a submanifold of middle dimension, and $F: L \rightarrow M$ be the inclusion. Let $J_{\alpha}, \alpha=1,2,3$ be parallel complex structures such that $J_{1} J_{2}=J_{3}=-J_{2} J_{1}$. Then any $J=\sum_{\alpha=1}^{3} a_{\alpha} J_{\alpha}$ with constant $a=\left(a_{1}, a_{2}, a_{3}\right) \in \mathbb{S}^{2}$ is also a parallel complex structure. Let $\mathcal{J}$ be the set of all $J \in \Gamma\left(F^{-1}(\operatorname{End}(T M))\right.$ such that

$$
J(p)=\sum_{\alpha=1}^{3} a_{\alpha}(p) J_{\alpha} \quad \text { with } \quad\left(a_{1}(p), a_{2}(p), a_{3}(p)\right) \in \mathbb{S}^{2} .
$$

Note that $\mathcal{J}$ can be identified with the set of smooth maps $a: L \rightarrow \mathbb{S}^{2}$.

For any $J \in \mathcal{J}$ we follow the definitions in [11] to define

(1) the 2-form

$$
\omega(J)(\cdot, \cdot)=\bar{g}(J \cdot, \cdot),
$$

(2) an operator $N(J): T_{p} L \rightarrow\left(T_{p} L\right)^{\perp}$ by

$$
N(J) v:=(J v)^{\perp} \text {, and }
$$

(3) a tensor on $L$ :

$$
h(J)(u, v, w):=-\bar{g}\left(N(J)(u), \bar{\nabla}_{v} w\right)=\bar{g}\left(\bar{\nabla}_{v}(N(J)(u)), w\right) .
$$

In order to simplify notations, we will write $\omega, N$ and $h$ for $\omega(J), N(J)$, and $h(J)$, respectively, if the dependence on $J$ is clear. And we will reserve the notations $\omega_{\alpha}, N_{\alpha}$, and $h_{\alpha}$ for $\omega\left(J_{\alpha}\right), N\left(J_{\alpha}\right)$, and $h\left(J_{\alpha}\right)$, respectively, once a choice of $\left\{J_{\alpha}\right\}_{\alpha=1}^{3}$ is fixed. It is then obvious that $\omega=\sum a_{\alpha} \omega\left(J_{\alpha}\right)$, $N=\sum a_{\alpha} N\left(J_{\alpha}\right)$, and $h=\sum a_{\alpha} h\left(J_{\alpha}\right)$; and the construction is independent on the choice of the orthogonal set of parallel complex structures $J_{\alpha}, \alpha=1,2,3$.

Let $x^{i}, i=1, \ldots, 2 n$, and $y^{\kappa}, \kappa=1, \ldots, 4 n$ be local coordinates on $L$ and $M$, respectively. Let $e_{i}=d F\left(\partial_{x_{i}}\right)$. Then $\left\{e_{i}\right\}$ is a basis for $T L$ in $F^{-1} T M$. And we will denote $h_{i j k}=h(J)_{i j k}=h(J)\left(e_{i}, e_{j}, e_{k}\right)$ for $J \in \mathcal{J}$ and $h_{\alpha, i j k}=h_{\alpha}\left(e_{i}, e_{j}, e_{k}\right)$. Similarly, we will write $\omega_{\alpha, i j}=\omega_{\alpha}\left(e_{i}, e_{j}\right)$.

Suppose that $N(J)$ is an isomorphism in a coordinate neighborhood. Then $\eta(J)_{i j}:=$ $\bar{g}\left(N(J)\left(e_{i}\right), N(J)\left(e_{j}\right)\right)$ is invertible and we will denote the inverse by $\eta(J)^{i j}$. As before, we will write $\eta_{i j}$ and $\eta^{i j}$ for the purpose of simplifying notations. 
Most of the equations used in [11] are still valid for general $J \in \mathcal{J}$. In particular, we have

$$
\begin{aligned}
N\left(e_{i}\right) & =J e_{i}-\sum_{j=1}^{2 n} \omega_{i}^{j} e_{j}, \\
\eta_{i j} & =g_{i j}+\omega_{i}^{k} \omega_{k j} . \\
\bar{\nabla}_{e_{k}} e_{j} & =\Gamma_{k j}^{n} e_{n}-\eta^{m n} h_{m k j} N\left(e_{n}\right) .
\end{aligned}
$$

Note that only quantities in the second term on the right-hand side of the Equation (2.3) depends on $J$. We also have

$$
\begin{aligned}
\nabla_{l} h_{i k j}-\nabla_{k} h_{i l j}= & \bar{R}_{\underline{i} j k l}+\eta^{m n} \omega_{n}^{s}\left(h_{m l j} h_{s k i}-h_{m k j} h_{s l i}\right) \\
& +\eta^{m n} \omega_{i}^{s}\left(h_{m k j} h_{n l s}-h_{m l j} h_{n k s}\right),
\end{aligned}
$$

where $\bar{R}_{\underline{i} j k l}=\bar{g}\left(\bar{R}\left(e_{k}, e_{l}\right) e_{j}, N\left(e_{i}\right)\right)$, and

$$
R_{i j k l}=\bar{R}_{i j k l}+\eta^{m n}\left(h_{m i k} h_{n j l}-h_{m i l} h_{n j k}\right) .
$$

However, when $\omega$ is no longer parallel or closed, other equations have to be modified.

Proposition 2.1. For any orthogonal set of parallel complex structures $\left\{J_{\alpha}\right\}_{\alpha=1}^{3}$ and any $J=$ $\sum_{\alpha=1}^{3} a_{\alpha} J_{\alpha}$, the corresponding $h=h(J)$ and $\omega=\omega(J)$ satisfy

$$
h_{k i j}=h_{i k j}+\nabla_{j} \omega_{i k}-\sum_{\alpha=1}^{3}\left(\nabla_{j} a_{\alpha}\right) \omega_{\alpha, i k} .
$$

Proof. The proof is straightforward by the corresponding proposition of [11]. As each $J_{\alpha}$ is parallel,

$$
\begin{aligned}
h_{k i j} & =\sum_{\alpha} a_{\alpha} h_{\alpha, k i j} \\
& =\sum_{\alpha} a_{\alpha}\left(h_{\alpha, i k j}+\nabla_{j} \omega_{\alpha, i k}\right) \\
& =h_{i k j}+\sum_{\alpha} a_{\alpha} \nabla_{j} \omega_{\alpha, i k} \\
& =h_{i k j}+\nabla_{j} \omega_{i k}-\sum_{\alpha}\left(\nabla_{j} a_{\alpha}\right) \omega_{\alpha, i k} .
\end{aligned}
$$

Proposition 2.2. For any $J \in \mathcal{J}$, the corresponding 2-form $\omega=\omega(J)$ satisfies

$$
\nabla_{k} \nabla_{j} \omega_{l i}-\nabla_{l} \nabla_{j} \omega_{k i}=\nabla_{j} \nabla_{i} \omega_{l k}+R_{i l j}^{s} \omega_{k s}+R_{i j k}^{s} \omega_{l s}-R_{j k l}^{s} \omega_{s i}+\nabla_{j}(d \omega)_{l i k}
$$

Proof. The proof is exactly the same as in [11]. The only modification is that $\omega$ is not closed, so the last term does not vanish in general.

Proposition 2.3. For any orthogonal set of parallel complex structures $\left\{J_{\alpha}\right\}_{\alpha=1}^{3}$ and any $J=$ 
$\sum_{\alpha=1}^{3} a_{\alpha} J_{\alpha}$, we have

$$
\begin{aligned}
\nabla_{l} h_{k i j}-\nabla_{k} h_{l i j}= & \bar{R}_{\underline{i} j k l}+\nabla_{j} \nabla_{i} \omega_{l k}+\omega_{i}^{s} \bar{R}_{s j k l}+\omega_{k}^{s} R_{s i l j}+\omega_{l}^{s} R_{s i j k} \\
& +\eta^{m n} \omega_{n}^{s}\left(h_{m l j} h_{s k i}-h_{m k j} h_{s l i}\right) \\
& +\sum_{\alpha}\left(\nabla_{i} \nabla_{j} a_{\alpha}\right) \omega_{\alpha, k l} \\
& +\sum_{\alpha}\left(\nabla_{i} a_{\alpha} \nabla_{j} \omega_{\alpha, k l}+\nabla_{j} a_{\alpha} \nabla_{i} \omega_{\alpha, k l}\right) \\
& +\sum_{\alpha}\left(\nabla_{l} a_{\alpha} \nabla_{j} \omega_{\alpha, i k}+\nabla_{k} a_{\alpha} \nabla_{j} \omega_{\alpha, l i}\right)
\end{aligned}
$$

Proof. By Proposition 2.1,

$$
\begin{aligned}
\nabla_{l} h_{k i j}-\nabla_{k} h_{l i j}= & \nabla_{l}\left(h_{i k j}+\nabla_{j} \omega_{i k}-\sum_{\alpha}\left(\nabla_{j} a_{\alpha}\right) \omega_{\alpha, i k}\right) \\
& -\nabla_{k}\left(h_{i l j}+\nabla_{j} \omega_{i l}-\sum_{\alpha}\left(\nabla_{j} a_{\alpha}\right) \omega_{\alpha, i l}\right) \\
= & \left(\nabla_{l} h_{i k j}-\nabla_{k} h_{i l j}\right)+\left(\nabla_{k} \nabla_{j} \omega_{l i}-\nabla_{l} \nabla_{j} \omega_{k i}\right) \\
& +\sum_{\alpha}\left[\nabla_{k}\left(\left(\nabla_{j} a_{\alpha}\right) \omega_{\alpha, i l}\right)-\nabla_{l}\left(\left(\nabla_{j} a_{\alpha}\right) \omega_{\alpha, i k}\right)\right] .
\end{aligned}
$$

The first two terms can be handled using Proposition 2.2 and Equation (2.4) as in [11] and we obtain

$$
\begin{aligned}
\nabla_{l} h_{k i j}-\nabla_{k} h_{l i j}= & \bar{R}_{\underline{i j k l}}+\nabla_{j} \nabla_{i} \omega_{l k}+\omega_{i}^{s} \bar{R}_{s j k l}+\omega_{k}^{s} R_{s i l j}+\omega_{l}^{s} R_{s i j k} \\
& +\eta^{m n}\left(h_{m l j} h_{s k i}-h_{m k j} h_{s l i}\right) \\
& +\nabla_{j}(d \omega)_{l i k}+\sum_{\alpha}\left[\nabla_{k}\left(\left(\nabla_{j} a_{\alpha}\right) \omega_{\alpha, i l}\right)-\nabla_{l}\left(\left(\nabla_{j} a_{\alpha}\right) \omega_{\alpha, i k}\right)\right] .
\end{aligned}
$$

Since $\omega_{\alpha}$ are closed for all $\alpha=1,2,3$, we have

$$
\begin{aligned}
\nabla_{j}(d \omega)_{l i k}= & \sum_{\alpha} \nabla_{j}\left(d a_{\alpha} \wedge \omega_{\alpha}\right)_{l i k} \\
= & \sum_{\alpha} \nabla_{j}\left[\left(\nabla_{l} a_{\alpha}\right) \omega_{\alpha, i k}-\left(\nabla_{i} a_{\alpha}\right) \omega_{\alpha, l k}+\left(\nabla_{k} a_{\alpha}\right) \omega_{\alpha, l i}\right] \\
= & \sum_{\alpha}\left[\left(\nabla_{j} \nabla_{l} a_{\alpha}\right) \omega_{\alpha, i k}+\left(\nabla_{j} \nabla_{i} a_{\alpha}\right) \omega_{\alpha, k l}+\left(\nabla_{j} \nabla_{k} a_{\alpha}\right) \omega_{\alpha, l i}\right] \\
& +\sum_{\alpha}\left[\left(\nabla_{l} a_{\alpha}\right) \nabla_{j} \omega_{\alpha, i k}+\left(\nabla_{i} a_{\alpha}\right) \nabla_{j} \omega_{\alpha, k l}+\left(\nabla_{k} a_{\alpha}\right) \nabla_{j} \omega_{\alpha, l i}\right] .
\end{aligned}
$$

On the other hand,

$$
\begin{aligned}
\sum_{\alpha} & {\left[\nabla_{k}\left(\left(\nabla_{j} a_{\alpha}\right) \omega_{\alpha, i l}\right)-\nabla_{l}\left(\left(\nabla_{j} a_{\alpha}\right) \omega_{\alpha, i k}\right)\right] } \\
= & \sum_{\alpha}\left[\left(\nabla_{k} \nabla_{j} a_{\alpha}\right) \omega_{\alpha, i l}+\nabla_{j} a_{\alpha} \nabla_{k} \omega_{\alpha, i l}\right] \\
& \quad-\sum_{\alpha}\left[\left(\nabla_{l} \nabla_{j} a_{\alpha}\right) \omega_{\alpha, i k}+\nabla_{j} a_{\alpha} \nabla_{l} \omega_{\alpha, i k}\right] \\
= & \sum_{\alpha}\left[\left(\nabla_{k} \nabla_{j} a_{\alpha}\right) \omega_{\alpha, i l}-\left(\nabla_{l} \nabla_{j} a_{\alpha}\right) \omega_{\alpha, i k}+\nabla_{j} a_{\alpha} \nabla_{i} \omega_{\alpha, k l}\right] .
\end{aligned}
$$


Therefore, the extra terms in the last line of Equation (2.6) can be written as

$$
\begin{aligned}
& \nabla_{j}(d \omega)_{l i k}+\sum_{\alpha}\left[\nabla_{k}\left(\left(\nabla_{j} a_{\alpha}\right) \omega_{\alpha, i l}\right)-\nabla_{l}\left(\left(\nabla_{j} a_{\alpha}\right) \omega_{\alpha, i k}\right)\right] \\
& =\sum_{\alpha}\left(\nabla_{j} \nabla_{i} a_{\alpha}\right) \omega_{\alpha, k l}+\sum_{\alpha}\left(\nabla_{i} a_{\alpha} \nabla_{j} \omega_{\alpha, k l}+\nabla_{j} a_{\alpha} \nabla_{i} \omega_{\alpha, k l}\right) \\
& \quad+\sum_{\alpha}\left(\nabla_{l} a_{\alpha} \nabla_{j} \omega_{\alpha, i k}+\nabla_{k} a_{\alpha} \nabla_{j} \omega_{\alpha, i l}\right),
\end{aligned}
$$

which is the desired result.

\section{Mean curvature flow}

In this section, we are going to prove that hyper-Lagrangian submanifolds are preserved under mean curvature flow. Our proof is similar to that of Smoczyk [11]. However, the defining almost complex structure $J$ of the hyper-Lagrangian submanifold and the 2-forms here corresponding the symplectic form are no longer parallel or closed. This introduces a lot of extra terms and a need of an estimate of the covariant derivative of the corresponding symplectic form. Moreover, unlike the situation in [11], the harmonic map heat flow equation is not just a consequence of the mean curvature flow, it will also be used to obtain the necessary estimate.

\subsection{Mean curvature flow}

As in [11], the assumption that $N$ is an isomorphism ensures that the mean curvature vector of $L$ can be written as

$$
H=-\eta^{m n} g^{k l} h_{m k l} N\left(e_{n}\right) .
$$

From now on, the corresponding mean curvature form with respect to $J$ is defined by

$$
\left.\sigma_{H}:=H\right\lrcorner \omega
$$

which can be written in term of coordinates as follow

$$
\sigma_{H}=H_{i} d x^{i}:=g^{k l} h_{i k l} d x^{i} .
$$

Similarly, for $\alpha=1,2,3$, we write

$$
\left.\sigma_{H_{\alpha}}:=H\right\lrcorner \omega_{\alpha}=H_{\alpha, i} d x^{i} .
$$

In terms of the coefficients of $\sigma_{H}$, the mean curvature vector can be written as

$$
H=-\eta^{m n} H_{m} N\left(e_{n}\right) \text {. }
$$

Note that all the above depend on the $J \in \mathcal{J}$ except the mean curvature vector.

Now, we assume that $F: L \rightarrow M$ is deformed under the mean curvature flow

$$
\frac{d}{d t} F_{t}=H, \quad F_{0}=F
$$

which can be written as

$$
\frac{d}{d t} F_{t}=-\eta^{m n} H_{m} N\left(e_{n}\right), \quad F_{0}=F
$$


for any $J \in \mathcal{J}$. Suppose that the mean curvature flow exists for $t \in[0, T)$ and the metric at time $t$ is $g_{t}$. Let $a(t, p):[0, T) \times L \rightarrow \mathbb{S}^{2}$ be a smooth mapping and $J(t)=\sum_{\alpha} a_{\alpha}(t, p) J_{\alpha}$ be the corresponding deformation of the tensor $J$ along the mean curvature flow. We are going to calculate the deformation of the corresponding 2- form $\omega(t)$ on $L_{t}$.

Proposition 3.1. Suppose that $J(t)=\sum_{\alpha} a_{\alpha}(t, p) J_{\alpha}$ is a deformation of $J=J(0)$ in $\mathcal{J}$ along the mean curvature flow. Then the corresponding 2-form $\omega(t)=\omega(J(t))$ satisfies

$$
\begin{aligned}
\frac{d}{d t} \omega_{i j}= & \bar{R}_{\underline{s}}{ }^{s}{ }_{j i}+\Delta \omega_{i j} \\
& +\omega_{k}^{s} \bar{R}_{s}{ }^{k}{ }_{j i}+\omega_{j}^{s} R_{s}{ }^{k}{ }_{i k}+\omega_{i}^{s} \bar{R}_{s}{ }^{k} k j \\
& +\eta^{m n} \omega_{n}^{s}\left(h_{m i}{ }^{k} h_{s j k}-h_{m j}{ }^{k} h_{s i k}\right) \\
& +\sum_{\alpha}\left[\left(\partial_{t} a_{\alpha}-\triangle a_{\alpha}\right) \omega_{\alpha, i j}-2 \nabla_{k} a_{\alpha} \nabla_{k} \omega_{\alpha, i j}\right] \\
& +\sum_{\alpha}\left(h_{\alpha, k k j} \nabla_{i} a_{\alpha}-h_{\alpha, k k i} \nabla_{j} a_{\alpha}\right) .
\end{aligned}
$$

Proof. Since $\omega_{\alpha}$ are parallel, we have for $\alpha=1,2,3$ that

$$
\left.\frac{\partial}{\partial t} \omega_{\alpha, i j}=\left(d \sigma_{H_{\alpha}}\right)_{i j}+(H\lrcorner d \omega_{\alpha}\right)_{i j}=\left(d \sigma_{H_{\alpha}}\right)_{i j} .
$$

Hence,

$$
\begin{aligned}
\frac{d}{d t} \omega_{i j} & =\sum_{\alpha}\left[\frac{\partial a_{\alpha}}{\partial t} \omega_{\alpha, i j}+a_{\alpha}\left(d \sigma_{H_{\alpha}}\right)_{i j}\right] \\
& =\sum_{\alpha}\left[\frac{\partial a_{\alpha}}{\partial t} \omega_{\alpha, i j}+\left(d\left(a_{\alpha} \sigma_{H_{\alpha}}\right)\right)_{i j}-\left(d a_{\alpha} \wedge \sigma_{H_{\alpha}}\right)_{i j}\right] \\
& =\sum_{\alpha}\left[\frac{\partial a_{\alpha}}{\partial t} \omega_{\alpha, i j}-\left(d a_{\alpha} \wedge \sigma_{H_{\alpha}}\right)_{i j}\right]+\left(d \sigma_{H}\right)_{i j} .
\end{aligned}
$$

Using Proposition 2.3, we have

$$
\begin{aligned}
\left(d \sigma_{H}\right)_{i j}= & \bar{R}_{\underline{s}}^{s}{ }_{j i}+\Delta \omega_{i j} \\
& +\omega_{k}^{s} \bar{R}_{s}{ }^{k}{ }_{j i}+\omega_{j}^{s} R_{S}{ }^{k}{ }_{i k}+\omega_{i}^{s} \bar{R}_{s}{ }^{k} k j \\
& +\eta^{m n} \omega_{n}^{s}\left(h_{m i}{ }^{k} h_{s j k}-h_{m j}{ }^{k} h_{s i k}\right) \\
& +\sum_{\alpha}\left[\left(\triangle a_{\alpha}\right) \omega_{\alpha, j i}+2 \nabla_{k} a_{\alpha} \nabla_{k} \omega_{\alpha, j i}\right] \\
& +\sum_{\alpha}\left(\nabla_{i} a_{\alpha} \nabla_{k} \omega_{\alpha, k j}-\nabla_{j} a_{\alpha} \nabla_{k} \omega_{\alpha, k i}\right) .
\end{aligned}
$$

Therefore, together with

$$
\sum_{\alpha}\left(d a_{\alpha} \wedge \sigma_{H_{\alpha}}\right)_{i j}=\sum_{\alpha}\left[\left(\nabla_{i} a_{\alpha}\right) H_{\alpha, j}-\left(\nabla_{j} a_{\alpha}\right) H_{\alpha, i}\right]
$$


we have

$$
\begin{aligned}
\frac{d}{d t} \omega_{i j}= & \bar{R}_{\underline{s}}{ }^{s}{ }_{j i}+\Delta \omega_{i j} \\
& +\omega_{k}^{s} \bar{R}_{s}{ }^{k}{ }_{j i}+\omega_{j}^{s} R_{s}{ }^{k}{ }_{i k}+\omega_{i}^{s} \bar{R}_{s}{ }^{k} k j \\
& +\eta^{m n} \omega_{n}^{s}\left(h_{m i}{ }^{k} h_{s j k}-h_{m j}{ }^{k} h_{s i k}\right) \\
& +\sum_{\alpha}\left[\left(\partial_{t} a_{\alpha}-\triangle a_{\alpha}\right) \omega_{\alpha, i j}-2 \nabla_{k} a_{\alpha} \nabla_{k} \omega_{\alpha, i j}\right] \\
& +\sum_{\alpha}\left[\nabla_{i} a_{\alpha}\left(\nabla_{k} \omega_{\alpha, k j}-H_{\alpha, j}\right)-\nabla_{j} a_{\alpha}\left(\nabla_{k} \omega_{\alpha, k i}-H_{\alpha, i}\right)\right] .
\end{aligned}
$$

Finally, by Proposition 2.1, for each $\alpha=1,2,3$, we have

$$
H_{\alpha, i}=h_{\alpha, i k k}=h_{\alpha, k i k}+\nabla_{k} \omega_{\alpha, k i}
$$

Putting this in the above and note that $h_{\alpha, k i k}=h_{\alpha, k k i}$, we have proved the proposition.

Lemma 3.2. Let $a: L \rightarrow \mathbb{S}^{2}$ be the map corresponding to some $J \in \mathcal{J}$ with respect to an orthogonal set of complex structures $\left\{J_{\alpha}\right\}_{\alpha=1}^{3}$. Then we have

$$
\sum_{\alpha}\left(\nabla_{i} a_{\alpha}\right) h_{\alpha, k k j}=\eta^{l s} h_{l k j} \sum_{\alpha}\left[\left(a \times \nabla_{i} a\right)_{\alpha} \omega_{\alpha, k s}+\omega_{s}^{n}\left(\nabla_{i} a_{\alpha}\right) \omega_{\alpha, k n}\right]
$$

where $a \times \nabla_{i} a$ is the cross product of $a=\left(a_{\alpha}\right)$ and $\nabla_{i} a=\left(\nabla_{i} a_{\alpha}\right)$ by regarding them as vectors in 3-space.

Proof. By definition

$$
\begin{aligned}
h_{\alpha, k k j} & =-\bar{g}\left(N_{\alpha} e_{k}, \bar{\nabla}_{e_{k}} e_{j}\right) \\
& =-\bar{g}\left(\left(J_{\alpha} e_{k}\right)^{\perp}, \bar{\nabla}_{e_{k}} e_{j}\right) \\
& =-\bar{g}\left(J_{\alpha} e_{k},\left(\bar{\nabla}_{e_{k}} e_{j}\right)^{\perp}\right) \\
& =-\bar{g}\left(J_{\alpha} e_{k}, \eta^{l s} h_{l k j} N\left(e_{s}\right)\right) \\
& =-\eta^{l s} h_{l k j} \bar{g}\left(J_{\alpha} e_{k}, J e_{s}-\omega_{s}^{n} e_{n}\right) \\
& =\eta^{l s} h_{l k j}\left[\omega_{\alpha, k n} \omega_{s}^{n}-\bar{g}\left(J_{\alpha} e_{k}, J e_{s}\right)\right] \\
& =\eta^{l s} h_{l k j}\left[\omega_{\alpha, k n} \omega_{s}^{n}-\sum_{\beta} a_{\beta} \bar{g}\left(J_{\alpha} e_{k}, J_{\beta} e_{s}\right)\right] .
\end{aligned}
$$

Therefore

$$
\begin{aligned}
\sum_{\alpha}\left(\nabla_{i} a_{\alpha}\right) h_{\alpha, k k j}= & \eta^{l s} h_{l k j} \sum_{\alpha}\left(\nabla_{i} a_{\alpha}\right) \omega_{\alpha, k n} \omega_{s}^{n} \\
& -\eta^{l s} h_{l k j} \sum_{\alpha, \beta}\left(a_{\beta} \nabla_{i} a_{\alpha}\right) \bar{g}\left(J_{\alpha} e_{k}, J_{\beta} e_{s}\right)
\end{aligned}
$$




$$
\begin{aligned}
= & \eta^{l s} h_{l k j} \sum_{\alpha}\left(\nabla_{i} a_{\alpha}\right) \omega_{\alpha, k n} \omega_{s}^{n} \\
& -\eta^{l s} h_{l k j} \sum_{\alpha}\left(a_{\alpha} \nabla_{i} a_{\alpha}\right) \bar{g}\left(J_{\alpha} e_{k}, J_{\alpha} e_{s}\right) \\
& +\eta^{l s} h_{l k j} \sum_{\beta<\alpha}\left(a_{\alpha} \nabla_{i} a_{\beta}-a_{\beta} \nabla_{i} a_{\alpha}\right) \bar{g}\left(J_{\alpha} J_{\beta} e_{k}, e_{s}\right) \\
=\quad & \eta^{l s} h_{l k j} \sum_{\alpha}\left(\nabla_{i} a_{\alpha}\right) \omega_{\alpha, k n} \omega_{s}^{n} \\
& -\eta^{l s} h_{l k j} g_{k s} \sum_{\alpha}\left(a_{\alpha} \nabla_{i} a_{\alpha}\right) \\
& +\eta^{l s} h_{l k j} \sum_{\beta<\alpha}\left(a_{\alpha} \nabla_{i} a_{\beta}-a_{\beta} \nabla_{i} a_{\alpha}\right) \bar{g}\left(J_{\alpha} J_{\beta} e_{k}, e_{s}\right) .
\end{aligned}
$$

Using the fact that $\sum_{\alpha} a_{\alpha}^{2}=1$, we have $\sum_{\alpha} a_{\alpha} \nabla_{i} a_{\alpha}=0$ and hence

$$
\begin{aligned}
\sum_{\alpha}\left(\nabla_{i} a_{\alpha}\right) h_{\alpha, k k j}= & \eta^{l s} h_{l k j} \sum_{\alpha}\left(\nabla_{i} a_{\alpha}\right) \omega_{\alpha, k n} \omega_{s}^{n} \\
& +\eta^{l s} h_{l k j} \sum_{\beta<\alpha}\left(a_{\alpha} \nabla_{i} a_{\beta}-a_{\beta} \nabla_{i} a_{\alpha}\right) \bar{g}\left(J_{\alpha} J_{\beta} e_{k}, e_{s}\right) .
\end{aligned}
$$

This is the desired result.

Lemma 3.3. Let $\left\{J_{\alpha}\right\}_{\alpha=1}^{3}$ be an orthogonal set of complex structures of a hyperkähler manifold $\left(M^{4 n}, \bar{g}\right)$ such that $N_{2}=N\left(J_{2}\right)$ and $N_{3}=N\left(J_{3}\right)$ are isomorphisms at each point of a submanifold $L^{2 n} \subset M$. Then

$$
\begin{aligned}
\nabla_{i} \omega_{1, k l}= & -\eta_{3}{ }^{m n} h_{3, m i k} \omega_{2, l n}+\eta_{3}{ }^{m n} h_{3, m i k} \omega_{1, l j} \omega_{3, n}^{j} \\
& -\eta_{2}{ }^{m n} h_{2, m i l} \omega_{3, k n}-\eta_{2}{ }^{m n} h_{2, m i l} \omega_{1, k j} \omega_{2, n}^{j} .
\end{aligned}
$$

Moreover, if $L$ is compact, then there is a constant $C>0$ such that

$$
\left|\nabla \omega_{1}\right| \leq C \sqrt{\left|\omega_{2}\right|^{2}+\left|\omega_{3}\right|^{2}} .
$$

Proof. Let $e_{i}$ be an orthonormal basis of $T L$ in a neighborhood of a point $p$. Then by definition,

$$
\nabla_{i} \omega_{1, k l}=\left(\nabla_{e_{i}} \omega_{1}\right)\left(e_{k}, e_{l}\right)=e_{i}\left(\omega_{1}\left(e_{k}, e_{l}\right)\right)-\omega_{1}\left(\nabla_{e_{i}} e_{k}, e_{l}\right)-\omega_{1}\left(e_{k}, \nabla_{e_{i}} e_{l}\right) .
$$

Since $\bar{\omega}_{1}$ is parallel, we have

$$
\begin{aligned}
\nabla_{i} \omega_{1, k l} & =\bar{\omega}_{1}\left(\bar{\nabla}_{e_{i}} e_{k}, e_{l}\right)+\bar{\omega}_{1}\left(e_{k}, \bar{\nabla}_{e_{i}} e_{l}\right)-\bar{\omega}_{1}\left(\nabla_{e_{i}} e_{k}, e_{l}\right)-\bar{\omega}_{1}\left(e_{k}, \nabla_{e_{i}} e_{l}\right) \\
& =\bar{\omega}_{1}\left(\left(\bar{\nabla}_{e_{i}} e_{k}\right)^{\perp}, e_{l}\right)+\bar{\omega}_{1}\left(e_{k},\left(\bar{\nabla}_{e_{i}} e_{l}\right)^{\perp}\right) .
\end{aligned}
$$

Using $J_{1}=J_{2} J_{3}$,

$$
\begin{aligned}
\nabla_{i} \omega_{1, k l} & =\bar{g}\left(J_{2} J_{3}\left(\bar{\nabla}_{e_{i}} e_{k}\right)^{\perp}, e_{l}\right)+\bar{g}\left(J_{2} J_{3} e_{k},\left(\bar{\nabla}_{e_{i}} e_{l}\right)^{\perp}\right) \\
& =-\bar{g}\left(J_{2} e_{l}, J_{3}\left(\bar{\nabla}_{e_{i}} e_{k}\right)^{\perp}\right)-\bar{g}\left(J_{3} e_{k}, J_{2}\left(\bar{\nabla}_{e_{i}} e_{l}\right)^{\perp}\right) .
\end{aligned}
$$


Note that

$$
\left(\bar{\nabla}_{e_{i}} e_{k}\right)^{\perp}=-\eta_{2}^{m n} h_{2, m i k} N_{2}\left(e_{n}\right)=-\eta_{3}{ }^{m n} h_{3, m i k} N_{3}\left(e_{n}\right),
$$

we have

$$
\begin{aligned}
\nabla_{i} \omega_{1, k l} & =\bar{g}\left(J_{2} e_{l}, J_{3}\left(\eta_{3}{ }^{m n} h_{3, m i k} N_{3}\left(e_{n}\right)\right)\right)+\bar{g}\left(J_{3} e_{k}, J_{2}\left(\eta_{2}{ }^{m n} h_{2, m i l} N_{2}\left(e_{n}\right)\right)\right) \\
& =\eta_{3}{ }^{m n} h_{3, m i k} \bar{g}\left(J_{2} e_{l}, J_{3} N_{3}\left(e_{n}\right)\right)+\eta_{2}{ }^{m n} h_{2, m i l} \bar{g}\left(J_{3} e_{k}, J_{2} N_{2}\left(e_{n}\right)\right) .
\end{aligned}
$$

Then by the definition of the operator $N$, we have

$$
J_{2} N_{2}\left(e_{n}\right)=J_{2}\left(J_{2} e_{n}-\omega_{2, n}^{j} e_{j}\right)=-e_{n}-\omega_{2, n}^{j} J_{2} e_{j},
$$

and similarly for $J_{3}$. Putting this into the above, we conclude that

$$
\begin{aligned}
\nabla_{i} \omega_{1, k l}= & \eta_{3}{ }^{m n} h_{3, m i k}\left[\bar{g}\left(J_{2} e_{l},-e_{n}\right)-\omega_{3, n}^{j} \bar{g}\left(J_{2} e_{l}, J_{3} e_{j}\right)\right] \\
& +\eta_{2}{ }^{m n} h_{2, m i l}\left[\bar{g}\left(J_{3} e_{k},-e_{n}\right)-\omega_{2, n}^{j} \bar{g}\left(J_{3} e_{k}, J_{2} e_{j}\right)\right] \\
= & -\eta_{3}{ }^{m n} h_{3, m i k} \omega_{2, l n}-\eta_{3}{ }^{m n} h_{3, m i k} \omega_{1, l j} \omega_{3, n}^{j} \\
& -\eta_{2}{ }^{m n} h_{2, m i l} \omega_{3, k n}-\eta_{2}{ }^{m n} h_{2, m i l} \omega_{1, k j} \omega_{2, n}^{j} .
\end{aligned}
$$

This completes the proof of the first part of the lemma and the last part of the lemma follows immediately.

\subsection{Main theorems and harmonic map heat flow}

Theorem 3.4. Let $\left(M^{4 n}, \bar{g}\right)$ be a hyperkähler manifold. Let $L_{t} \subset M^{4 n}, t \in[0, T)$ for some $T>0$, be a family of middle dimensional submanifolds given by the mean curvature flow. Suppose that for each point $(t, p) \in L_{t}$, there is a parallel complex structure $J$ of $M$ such that, for all parallel complex structure $K$ of $M$ orthogonal to $J$,

$$
\left.\bar{g}(K \cdot, \cdot)\right|_{T_{p} L_{t}}=0,
$$

and that $J$ is smooth in $(t, p)$. Then the defining map $J=J(t, x)$ from $[0, T) \times L_{0}$ to $\mathbb{S}^{2}$, the space of parallel complex structures of $M$, satisfies the harmonic map heat flow equations with variable metric

$$
\partial_{t} J=\triangle_{t} J,
$$

where $\triangle_{t} J$ is the tension field of $J$ with respect to the induced metric $g_{t}$ on $L_{t}$.

Proof. Let $t_{o} \in[0, T)$ and $p$ be a point in $L_{t_{o}}$. Then $J_{1}=J\left(t_{o}, p\right)$ is a fixed parallel complex structure of $M$. We may complete this to an orthogonal set $\left\{J_{\alpha}\right\}_{\alpha=1}^{3}$, i.e., $J_{1} J_{2}=J_{3}=-J_{2} J_{1}$. With respect to this basis, we can use standard spherical coordinates on $\mathbb{S}^{2}$ to represent $J$ in a neighborhood of $\left(t_{o}, p\right) \in[0, T) \times L_{0}$ by

$$
J=\cos \theta \sin \varphi J_{1}+\sin \theta \sin \varphi J_{2}+\cos \varphi J_{3},
$$

where $\theta$ and $\varphi$ are functions of $(t, x)$ in a neighborhood of $\left(t_{0}, p\right)$. Then it is easy to see that

$$
K=-\sin \theta J_{1}+\cos \theta J_{2},
$$

and

$$
J K=-\cos \theta \cos \varphi J_{1}-\sin \theta \cos \varphi J_{2}+\sin \varphi J_{3}
$$


are complex structures orthogonal to $J$. Let's denote these three combinations by $J=\sum_{\alpha} a_{\alpha} J_{\alpha}$, $K=\sum_{\alpha} b_{\alpha} J_{\alpha}, J K=\sum_{\alpha} c_{\alpha} J_{\alpha}$ and write $a=\left(a_{1}, a_{2}, a_{3}\right)$ and so on. Then the map $J$ is represented by

$$
a(t, x)=(\cos \theta \sin \varphi, \sin \theta \sin \varphi, \cos \varphi) .
$$

By a straightforward calculation, we have

$$
\begin{aligned}
a\left(t_{o}, p\right) & =(1,0,0), \quad b\left(t_{o}, p\right)=(0,1,0), \quad c\left(t_{o}, p\right)=(0,0,1), \\
\nabla b\left(t_{o}, p\right) & =\left(-\left.\nabla \theta\right|_{\left(t_{o}, p\right)}, 0,0\right), \quad \nabla c\left(t_{o}, p\right)=\left(\left.\nabla \varphi\right|_{\left(t_{o}, p\right)}, 0,0\right), \\
\partial_{t} b\left(t_{o}, p\right) & =\left(-\left.\partial_{t} \theta\right|_{\left(t_{o}, p\right)}, 0,0\right), \quad \partial_{t} c\left(t_{o}, p\right)=\left(\left.\partial_{t} \varphi\right|_{\left(t_{o}, p\right)}, 0,0\right), \\
b \times\left.\nabla b\right|_{\left(t_{o}, p\right)} & =\left(0,0,\left.\nabla \theta\right|_{\left(t_{o}, p\right)}\right), \quad c \times\left.\nabla c\right|_{\left(t_{o}, p\right)}=\left(0,\left.\nabla \varphi\right|_{\left(t_{o}, p\right)}, 0\right), \\
\triangle b\left(t_{o}, p\right) & =\left.\left(-\Delta \theta,-|\nabla \theta|^{2}, 0\right)\right|_{\left(t_{o}, p\right)},
\end{aligned}
$$

and

$$
\triangle c\left(t_{o}, p\right)=\left.\left(\triangle \varphi, 2 \nabla \theta \cdot \nabla \varphi,-|\nabla \varphi|^{2}\right)\right|_{\left(t_{o}, p\right)} .
$$

In the above, $\nabla$ and $\triangle$ denote the gradient and the Laplacian with respect to the induced metric on $L_{t_{o}} ; b \times \nabla b$ and $c \times \nabla c$ the cross products by regarding $b, \nabla b, c$, and $\nabla c$ as 3-vectors.

By assumption, both $\bar{g}(K \cdot, \cdot)$ and $\bar{g}(J K \cdot, \cdot)$ vanish on $T_{x} L_{t}$ for any $(t, x)$ in a neighborhood of $\left(t_{o}, p\right)$. In particular, $\omega_{2}=\omega_{3}=0$ at $\left(t_{o}, p\right)$. Then by applying Proposition 3.1 to the 2-form $\bar{g}(K \cdot, \cdot)$, we have at the point $\left(t_{o}, p\right)$,

$$
\begin{aligned}
0= & {\left[\left(\partial_{t}-\triangle\right) b_{1}\right] \omega_{1, i j}-2 \nabla_{k} b_{1} \nabla_{k} \omega_{1, i j} } \\
& +\sum_{\alpha}\left(h_{\alpha, k k j} \nabla_{i} b_{\alpha}-h_{\alpha, k k i} \nabla_{j} b_{\alpha}\right) .
\end{aligned}
$$

Note that we have used the fact that $\sum_{s} \bar{R}_{\underline{s} j i}^{s}=0$ whenever $\bar{g}(K \cdot, \cdot) \equiv 0$ by the flatness of the Ricci curvature, see [11]. On the other hand, Lemmas 3.3 and 3.2 imply

$$
\nabla \omega_{1, i j}=0
$$

and

$$
\sum_{\alpha} h_{\alpha, k k j} \nabla_{i} b_{\alpha}=0
$$

Hence, we conclude that

$$
0=\left[\left(\partial_{t}-\triangle_{t}\right) b_{1}\right] \omega_{1, i j} .
$$

Since $\omega_{2}=\omega_{3}=0$, we have $\omega_{1} \neq 0$. Therefore,

$$
\left(\partial_{t}-\triangle_{t}\right) b_{1}=0,
$$

which is equivalent to

$$
\left(\partial_{t}-\triangle_{t}\right) \theta=0 \quad \text { at }\left(t_{o}, p\right) .
$$

Similarly, by applying Proposition 3.1, and Lemmas 3.3 and 3.2 to the symplectic form $\bar{g}(J K \cdot, \cdot)$, we have

$$
\left(\partial_{t}-\triangle_{t}\right) \varphi=0 \quad \text { at }\left(t_{o}, p\right) .
$$

All together, we have proved that $J$ satisfies

$$
\left(\partial_{t}-\triangle_{t}\right) J=0
$$

as a mapping from $[0, T) \times L_{0}$ to $\mathbb{S}^{2}$. 
Note that we have used the same notation to denote the Laplacian of functions and the tension field of a map. However, as we have seen, no confusion will be created.

Lemma 3.5. Suppose that $\left(M^{4 n}, \bar{g}\right)$ is a hyperkähler manifold and $L_{t} \subset M^{4 n}, t \in[0, T)$ for some $T>0$, is a family of compact middle dimensional submanifolds given by the mean curvature flow. Let $J=J(t, x) \in \mathcal{J}$ be a harmonic map heat flow along the mean curvature flow $L_{t}$, i.e., $\left(\partial_{t}-\triangle_{t}\right) J=0$; and let $K=K(t, p)$ be the parallel complex structure of $M$ corresponding to $J(t, p)$ constructed in the proof of Theorem 3.4. If for each point $(t, p) \in L_{t}$, $N(K), N(J K): T_{p} L_{t} \rightarrow\left(T_{p} L_{t}\right)^{\perp}$ are isomorphisms, then there is a constant $c>0$ such that

$$
\frac{\partial}{\partial t}|\Omega|^{2} \leq \triangle_{t}|\Omega|^{2}+c|\Omega|^{2},
$$

where $\Omega(\cdot, \cdot)=\bar{g}(K \cdot, \cdot)+i \bar{g}(J K \cdot, \cdot)$.

Proof. Lets fix a point $\left(t_{0}, p\right)$ and use the same notations and conventions as in the proof of the Theorem 3.4. Then as in [11], we use the flatness of the Ricci curvature, Proposition 3.1, and Lemma 3.2 to deduce that, there is a constant $A_{1}>0$ such that

$$
\begin{aligned}
\frac{\partial}{\partial t}|\omega(K)|^{2} \leq & A_{1}|\omega(K)|^{2}+2\langle\omega(K), \Delta \omega(K)\rangle \\
& +2 \sum_{\alpha}\left[\left(\partial_{t} b_{\alpha}-\triangle b_{\alpha}\right)\left\langle\omega_{\alpha}, \omega(K)\right\rangle-2 \nabla_{k} b_{\alpha}\left\langle\nabla_{k} \omega_{\alpha}, \omega(K)\right\rangle\right] \\
& +2 \eta(K)^{l s} h(K)_{l k j} \sum_{\alpha}\left(b \times \nabla_{i} b\right)_{\alpha}\left\langle\omega_{\alpha}, \omega(K)\right\rangle,
\end{aligned}
$$

where $b \times \nabla_{i}$ is the cross product by regarding $b$ and $\nabla b$ as 3-vectors.

Evaluating at $\left(t_{0}, p\right)$, we have

$$
\begin{aligned}
\left(\partial_{t}-\triangle\right)|\omega(K)|^{2} \leq & A_{2}\left|\omega_{2}\right|^{2}+2|\nabla \theta|^{2}\left|\omega_{2}\right|^{2} \\
& +4 \nabla_{k} \theta\left\langle\nabla_{k} \omega_{1}, \omega_{2}\right\rangle+2 \eta_{2}^{l s} h_{2, l k j} \nabla_{i} \theta\left\langle\omega_{3}, \omega_{2}\right\rangle,
\end{aligned}
$$

for some constant $A_{2}>0$. Since $L_{t}$ are compact, we can apply the last part of Lemma 3.3 to conclude that there is a constant $A_{3}>0$ such that

$$
\left(\partial_{t}-\Delta\right)|\omega(K)|^{2} \leq A_{3}\left(\left|\omega_{2}\right|^{2}+\left|\omega_{3}\right|^{2}\right) .
$$

Similarly, we have at the point $\left(t_{0}, p\right)$,

$$
\begin{aligned}
\left(\partial_{t}-\triangle\right)|\omega(J K)|^{2} \leq & B_{2}\left|\omega_{3}\right|^{2}-4 \nabla \theta \cdot \nabla \varphi\left\langle\omega_{2}, \omega_{3}\right\rangle \\
& +2|\nabla \varphi|^{2}\left|\omega_{3}\right|^{2}-4 \nabla_{k} \varphi\left\langle\nabla_{k} \omega_{1}, \omega_{3}\right\rangle \\
& +2 \eta_{3}^{l s} h_{3, l k j} \nabla_{i} \varphi\left\langle\omega_{2}, \omega_{3}\right\rangle
\end{aligned}
$$

for some constant $B_{2}>0$. Hence, there exists a constant $B_{3}>0$ such that

$$
\left(\partial_{t}-\Delta\right)|\omega(J K)|^{2} \leq B_{3}\left(\left|\omega_{2}\right|^{2}+\left|\omega_{3}\right|^{2}\right) .
$$

Combining these two inequalities, we have shown that

$$
\left(\partial_{t}-\Delta\right)|\Omega|^{2} \leq c|\Omega|^{2}
$$

with $c=A_{3}+B_{3}$. 
Next, we prove the main theorem of this section, namely, the mean curvature flow preserves the hyper-Lagrangian condition.

Theorem 3.6. Let $\left(M^{4 n}, \bar{g}\right)$ be a hyperkähler manifold. Let $L_{t} \subset M^{4 n}, t \in[0, T)$ for some $T>0$, be a family of middle dimensional submanifolds given by the mean curvature flow. Suppose that $L_{0} \subset M$ is hyper-Lagrangian. Then $L_{t}$ is hyper-Lagrangian for all $t \in[0, T)$.

Proof. Consider the initial value problem for the generalized harmonic map heat flow to $\mathbb{S}^{2}$ along the mean curvature flow:

$$
\left\{\begin{array}{l}
\left(\partial_{t}-\triangle_{t}\right) \Theta=0, \quad \text { on } \quad(0, T) \times L_{0} \\
\Theta(0, x)=J(x) .
\end{array}\right.
$$

The short time existence implies that there is a $T_{1} \in(0, T)$ such that solution $\Theta(t, x)=J(t, x)$ exists for $t \in\left[0, T_{1}\right)$. Recall that if $L_{0}$ is hyper-Lagrangian, then for each point $(0, p) \in L_{0}$, there is a parallel complex structure $J$ of $M$ such that, for all parallel complex structure $K$ of $M$ orthogonal to $J$,

$$
\left.\bar{g}(K \cdot, \cdot)\right|_{T_{p} L_{0}}=0 .
$$

So we can pick $K(t, x)$ satisfying the condition in the Lemma 3.5 and conclude that the corresponding complex 2 -form $\Omega$ satisfies

$$
\left\{\begin{array}{l}
\left(\partial_{t}-\Delta\right)|\Omega|^{2} \leq c|\Omega|^{2}, \quad \text { on }\left(0, T_{1}\right) \times L_{0} \\
|\Omega|^{2}(0, p) \equiv 0
\end{array}\right.
$$

Therefore maximum principle implies that $|\Omega|^{2} \equiv 0$, by the assumption that $L_{0}$ is hyperLagrangian. This proves the result for a short time.

Now using the same notation as in the proof of the theorem, we see that

$$
\bar{g}(K \cdot, \cdot) \equiv \bar{g}(J K \cdot, \cdot) \equiv 0
$$

along $L_{t}$. Therefore at any point $(t, p) \in\left[0, T_{1}\right) \times L_{0}$ and in the basis and coordinates chosen as in the proof of the theorem, we have

$$
\nabla\left(b_{\alpha} \omega_{\alpha}\right)=\nabla\left(c_{\alpha} \omega_{\alpha}\right)=0 .
$$

This implies, at the point $(t, p)$,

$$
\nabla b_{1} \omega_{1}+\nabla \omega_{2}=\nabla c_{1} \omega_{1}+\nabla \omega_{3}=0 .
$$

So

$$
|\nabla \theta| \leq\left|\nabla \omega_{2}\right| \quad \text { and } \quad|\nabla \varphi| \leq\left|\nabla \omega_{3}\right| .
$$

Noting that $\nabla_{j} \omega_{\alpha, i k}=h_{\alpha, k i j}-h_{\alpha, i k j}$ and $\left|h_{\alpha, k i j}\right| \leq\left|\bar{\nabla}_{e_{i}} e_{j}\right|$, we conclude that $|\nabla \theta|$ and $|\nabla \varphi|$ are bounded in terms of the geometry of mean curvature flow $L_{t}$ as long as it exists. Hence, if $T_{1}<T$, we can find subsequence of $J(t)$ converges to some element $J$ in $\mathcal{J}$ as $t \rightarrow T_{1}$. It is clear that $J$ enjoys the same property on $L_{T_{1}}$ as $J(t)$ on $L_{t}$, for $t<T_{1}$. This implies that $L_{T_{1}}$ is also hyper-Lagrangian.

In conclusion, we have proved that the set of $t \in[0, T)$ with hyper-Lagrangian $L_{t}$ is both open and closed in $[0, T)$. This, of course, implies that $L_{t}$ is hyper-Lagrangian for all $t \in[0, T)$. 


\section{Hyper-Lagrangian submanifolds}

Let $\left(L^{2 n}, g\right)$ be a hyper-Lagrangian submanifold in a hyperkähler manifold $\left(M^{4 n}, \bar{g}\right)$ with respect to some $J(x)$ in $\Lambda_{+, x}^{2}$ and $K(x): L \rightarrow \Lambda_{+, x}^{2}$ be a smooth map such that $K(x)$ is orthogonal to $J(x)$ for each $x \in L$. Then by the hyper-Lagrangian condition, we have

$$
\left.\omega_{K}\right|_{L}=0, \quad \forall x \in L .
$$

Suppose that $\left\{e_{i}\right\}_{1}^{2 n}$ is an orthonormal frame field for $T L$ around a point $x=p$ satisfying

$$
J e_{2 v-1}=e_{2 v} \quad \text { for } \quad v=1, \ldots, n,
$$

then $\left\{f_{i}=K e_{i}\right\}_{i=1}^{2 n}$ is an orthonormal frame field for the normal bundle satisfying

$$
J f_{2 v-1}=-f_{2 v}, \quad \text { for } \quad v=1, \ldots, n .
$$

For the frame $\left\{e_{i}, f_{i}\right\}$, we denote

$$
\begin{cases}e_{v}^{\prime}=\frac{1}{2}\left(e_{2 v-1}-i e_{2 v}\right), & e_{v}^{\prime \prime}=\frac{1}{2}\left(e_{2 v-1}+i e_{2 v}\right), \\ f_{v}^{\prime}=\frac{1}{2}\left(f_{2 v-1}+i f_{2 v}\right), & f_{v}^{\prime \prime}=\frac{1}{2}\left(f_{2 v-1}-i f_{2 v}\right) .\end{cases}
$$

Then it is obvious that

$$
\begin{aligned}
& \begin{cases}J e_{v}^{\prime}=i e_{v}^{\prime}, & J e_{v}^{\prime \prime}=-i e_{v}^{\prime \prime}, \\
J f_{v}^{\prime}=i f_{v}^{\prime}, & J f_{v}^{\prime \prime}=-i f_{v}^{\prime \prime},\end{cases} \\
& \begin{cases}K e_{v}^{\prime}=f_{v}^{\prime \prime}, & K e_{v}^{\prime \prime}=f_{v}^{\prime}, \\
K f_{v}^{\prime}=-e_{v}^{\prime \prime}, & K f_{v}^{\prime \prime}=-e_{v}^{\prime} .\end{cases}
\end{aligned}
$$

The frame fields $\left\{e_{\nu}^{\prime}, f_{\mu}^{\prime}\right\}$ defined in the above will be referred as the canonical frame adapted to $(J, K)$ around the point $p$.

Correspondingly, we set

$$
\begin{cases}\phi_{v}=\zeta_{2 v-1}+i \zeta_{2 v}, & \bar{\phi}_{v}=\zeta_{2 v-1}-i \zeta_{2 v} \\ \psi_{v}=\varphi_{2 v-1}-i \varphi_{2 v}, & \bar{\psi}_{v}=\varphi_{2 v-1}+i \varphi_{2 v}\end{cases}
$$

for the frame $\left\{\zeta_{1}, \ldots, \zeta_{2 n}, \varphi_{1}, \ldots, \varphi_{2 n}\right\}$ dual to $\left\{e_{1}, \ldots, e_{2 n}, f_{1} \ldots, f_{2 n}\right\}$. Then the $J$-holomorphic symplectic form is given by

$$
\Omega_{J}=\omega_{J K}-i \omega_{K}=-i \sum_{\nu=1}^{n} \phi_{\nu} \wedge \psi_{\nu}
$$

and the structural equations implies

$$
\left\{\begin{array}{l}
d \zeta_{i}=\sum_{j=1}^{2 n} \zeta_{i j} \wedge \zeta_{j}-\sum_{j=1}^{2 n} \Psi_{j i} \wedge \varphi_{j} \\
d \varphi_{i}=\sum_{j=1}^{2 n} \Psi_{i j} \wedge \zeta_{j}+\sum_{j=1}^{2 n} \varphi_{i j} \wedge \varphi_{j}
\end{array}\right.
$$

for some 1-forms $\zeta_{i j}, \Psi_{i j}$, and $\varphi_{i}$ satisfying

$$
\zeta_{i j}+\zeta_{j i}=0 \text { and } \varphi_{i j}+\varphi_{j i}=0 .
$$


Along the submanifold $L$, there exist $h_{i j k}$, symmetric in $j, k$, such that

$$
\Psi_{i j}=\sum_{k=1}^{2 n} h_{i j k} \zeta_{k}
$$

It is clear that if we choose a normal coordinates around the point $p$, then the $h_{i j k}$ defined here coincides with the $h(K)_{i j k}$ defined in the previous section at the point $p$. Finally, the mean curvature vector is of course given by

$$
H=-\sum_{i=1}^{2 n}\left(\sum_{k=1}^{2 n} h_{i k k}\right) f_{i}
$$

From the above, the covariant derivatives of the orthonormal frames are given by

$$
\left\{\begin{array}{l}
\bar{\nabla} e_{i}=\sum_{j=1}^{2 n} \zeta_{i j} \otimes e_{j}-\sum_{j=1}^{2 n} \Psi_{j i} \otimes f_{j} \\
\bar{\nabla} f_{i}=\sum_{j=1}^{2 n} \Psi_{i j} \otimes e_{j}+\sum_{j=1}^{2 n} \varphi_{i j} \otimes f_{j},
\end{array}\right.
$$

where $\zeta_{i j}, \Psi_{i j}$, and $\varphi_{i j}$ are 1-forms. If we set

$$
\left\{\begin{array}{l}
\mathcal{A}_{\mu v}=\frac{1}{2}\left[\left(\zeta_{2 \mu-1,2 v-1}+\zeta_{2 \mu, 2 v}\right)-i\left(\zeta_{2 \mu, 2 v-1}-\zeta_{2 \mu-1,2 v}\right)\right], \\
\mathcal{B}_{\mu v}=\frac{1}{2}\left[\left(\zeta_{2 \mu-1,2 v-1}-\zeta_{2 \mu, 2 v}\right)-i\left(\zeta_{2 \mu, 2 v-1}+\zeta_{2 \mu-1,2 v}\right)\right], \\
\mathcal{C}_{\mu v}=\frac{1}{2}\left[\left(\Psi_{2 \mu-1,2 v-1}-\Psi_{2 \mu, 2 v}\right)-i\left(\Psi_{2 \mu, 2 v-1}+\Psi_{2 \mu-1,2 v}\right)\right], \\
\mathcal{D}_{\mu v}=\frac{1}{2}\left[\left(\Psi_{2 \mu-1,2 v-1}+\Psi_{2 \mu, 2 v}\right)-i\left(\Psi_{2 \mu-1,2 v}-\Psi_{2 \mu, 2 v-1}\right)\right], \\
\mathcal{E}_{\mu v}=\frac{1}{2}\left[\left(\varphi_{2 \mu-1,2 v-1}+\varphi_{2 \mu, 2 v}\right)+i\left(\varphi_{2 \mu, 2 v-1}-\varphi_{2 \mu-1,2 v}\right)\right], \\
\mathcal{F}_{\mu \nu}=\frac{1}{2}\left[\left(\varphi_{2 \mu-1,2 v-1}-\varphi_{2 \mu, 2 v}\right)+i\left(\varphi_{2 \mu, 2 v-1}+\varphi_{2 \mu-1,2 v}\right)\right],
\end{array}\right.
$$

for $\nu, \mu=1, \ldots, n$, then straightforward calculation using $\zeta_{i j}+\zeta_{j i}=0$ and $\varphi_{a b}+\varphi_{b a}=0$ shows for any $\mu, v=1, \ldots, n$,

$$
\left\{\begin{array}{l}
\bar{\nabla} e_{\nu}^{\prime}=\sum_{\mu}\left(\mathcal{A}_{v \mu} \otimes e_{\mu}^{\prime}+\mathcal{B}_{v \mu} \otimes e_{\mu}^{\prime \prime}\right)-\sum_{\mu}\left(\mathcal{C}_{\mu \nu} \otimes f_{\mu}^{\prime}+\mathcal{D}_{\mu \nu} \otimes f_{\mu}^{\prime \prime}\right), \\
\bar{\nabla} f_{v}^{\prime}=\sum_{\mu}\left(\overline{\mathcal{C}_{\nu \mu}} \otimes e_{\mu}^{\prime}+\mathcal{D}_{\nu \mu} \otimes e_{\mu}^{\prime \prime}\right)+\sum_{\mu}\left(\mathcal{E}_{v \mu} \otimes f_{\mu}^{\prime}+\mathcal{F}_{\nu \mu} \otimes f_{\mu}^{\prime \prime}\right)
\end{array}\right.
$$

and

$$
\begin{cases}\mathcal{A}_{\mu \nu}+\overline{\mathcal{A}}_{\mu \nu} & =\mathcal{B}_{v \mu}+\mathcal{B}_{\mu \nu}=0 \\ \mathcal{E}_{\mu \nu}+\overline{\mathcal{E}}_{v \mu} & =\mathcal{F}_{\mu \nu}+\mathcal{F}_{v \mu}=0\end{cases}
$$

In order to prove the compatibility condition, for any fixed $p \in L$, we set $J_{1}=J(p)$, $J_{2}=K(p)$, and $J_{3}=J_{1} J_{2}$. Then $\left\{J_{\alpha}\right\}_{\alpha=1}^{3}$ is an orthogonal set of parallel complex structures and they will be called the canonical basis adapted to $(J, K)$ at $p$. At the point $p$, we have

$$
\begin{aligned}
& J_{1}=\sum_{v=1}^{n}\left(e_{2 v-1} \wedge e_{2 v}-f_{2 v-1} \wedge f_{2 v}\right) \\
& J_{2}=\sum_{v=1}^{n}\left(e_{2 v-1} \wedge f_{2 v-1}+e_{2 v} \wedge f_{2 v}\right) \\
& J_{3}=\sum_{v=1}^{n}\left(f_{2 v} \wedge e_{2 v-1}-f_{2 v-1} \wedge e_{2 v}\right)
\end{aligned}
$$


in the canonical identification between 2-forms and 2-vectors.

Now we can state the following.

Theorem 4.1. Let $L^{2 n}$ be a hyper-Lagrangian in a hyperkähler manifold $M^{4 n}$ with defining map $J$. Let $K: L \rightarrow \mathbb{S}^{2}$ be any smooth map orthogonal to $J$ at every point of $L$. Then for any fixed point $p \in L$, the coefficient 1-forms of the structural equations for the canonical frame adapted to $(J, K)$ around $p$ satisfy the following conditions at the point $p$ :

$$
\begin{aligned}
\mathcal{A}_{\mu \nu}+\overline{\mathcal{A}}_{\mu \nu} & =0 \\
\mathcal{B}_{v \mu} & =0 \\
\mathcal{C}_{v \mu} & =\mathcal{C}_{\mu \nu}, \\
\mathcal{D}_{v \mu} & =\frac{1}{2} \delta_{v \mu}\left(d a_{3}+i d a_{2}\right), \\
\mathcal{E}_{\mu \nu}+\overline{\mathcal{E}}_{v \mu} & =0 \\
\mathcal{F}_{v \mu} & =0 \\
\mathcal{E}_{v \mu} & =\overline{\mathcal{A}_{v \mu}}+i \delta_{v \mu} d b_{3},
\end{aligned}
$$

where $a_{\alpha}$ and $b_{\alpha}$ are, respectively, the components of $J$ and $K$ with respect to the canonical basis adapted to $(J, K)$ at $p$.

Moreover, the differential of $J$ at $p$ as a map into $\mathbb{S}^{2}$ is given by

$$
d J_{p}=2\left(\operatorname{Im} \mathcal{D}_{v v} J_{2}+\operatorname{Re} \mathcal{D}_{v v} J_{3}\right) \quad \text { for any } \quad v=1, \ldots, n .
$$

Proof. We note that (4.3) and (4.7) are just (4.2). To prove the other conditions, we differentiate $J e_{v}^{\prime}=i e_{v}^{\prime}$ to obtain

$$
(\nabla J) e_{v}^{\prime}+J\left(\nabla e_{v}^{\prime}\right)=i \nabla e_{v}^{\prime}
$$

for all $v=1, \ldots, n$. Let $J_{\alpha}$ be the canonical basis adapted to $(J, K)$ at $p$, and $a_{\alpha}, b_{\alpha}$ be the coefficients of $J=\sum_{\alpha} a_{\alpha} J_{\alpha}$ and $K=\sum_{\alpha} b_{\alpha} J_{\alpha}$, respectively. Then by the hyper-Lagrangian condition, $\nabla J$ at the point $p$ is a linear combination of $J_{2}=K(p)$ and $J_{3}$. Therefore, we have the following.

$$
\nabla J=d a_{2} J_{2}+d a_{3} J_{3}
$$

at the point $p$. Putting this and the structural equations in the above equation, we obtain at the point $p$,

$$
\left(d a_{2}-i d a_{3}\right) f_{v}^{\prime \prime}=2 i \mathcal{B}_{\nu \mu} e_{\mu}^{\prime \prime}-2 i \mathcal{D}_{\nu \mu} f_{\mu}^{\prime \prime},
$$

which implies the Equations (4.6) and (4.4).

Similarly, applying the same argument to $J f_{v}^{\prime}=i f_{v}^{\prime}$ and $K e_{v}^{\prime \prime}=f_{v}^{\prime}$, we obtain (4.6) again and all other Equations (4.8), (4.5), (4.9) together with the identity $d a_{2}+d b_{1}=0$ at $p$. The last identity is automatically satisfied by the definition of $a_{\alpha}$ and $b_{\alpha}$.

The final part is an obvious conclusion of (4.6) and the proof is completed.

The theorem indicates that hyper-Lagrangian is a strong condition which imposes a lot of restrictions on the structural equations. As a consequence, we have the following.

Corollary 4.2. Let $L^{2 n}$ be a hyper-Lagrangian in a hyperkähler manifold $M^{4 n}$ and for any $p \in L, J(p)$ be the almost complex structure at $p$ such that $\left.\Omega_{J}(p)\right|_{T_{p} L}=0$. Then $J$ induces an integrable Kähler structure on $L$ with holomorphic normal bundle. 
Proof. From Theorem 4.1, we have

$$
\left\{\begin{array}{l}
\nabla e_{\nu}^{\prime}=\left(\bar{\nabla} e_{\nu}^{\prime}\right)^{T}=\sum_{\mu} \mathcal{A}_{\mu \nu} \otimes e_{\mu}^{\prime}, \\
\nabla^{\perp} f_{v}^{\prime}=\left(\bar{\nabla} f_{v}^{\prime}\right)^{\perp}=\sum_{\mu} \mathcal{E}_{v \mu} \otimes f_{\mu}^{\prime} .
\end{array}\right.
$$

The conclusions follow immediately.

In the neighborhood of the point $p$, it is more convenient to use the following local representation of the map $J$

$$
\Theta(x)=\frac{a_{1}(x)+i a_{2}(x)}{1-a_{3}(x)}
$$

via stereographic projection. Then the theorem implies that at the point $p$,

$$
\mathcal{D}_{\nu \mu}=\frac{1}{2} \delta_{\nu \mu} d \Theta
$$

Using $\Theta$, the conditions on the second fundamental form can be written as in the following.

Corollary 4.3. Let $L^{2 n}$ be a hyper-Lagrangian in a hyperkähler manifold $M^{4 n}$ with defining map $J$. Let $K: L \rightarrow \mathbb{S}^{2}$ be any smooth map orthogonal to $J$ at every point of $L$. Then for any fixed point $p \in L, \Psi_{2 \mu-1,2 v-1}, \Psi_{2 \mu, 2 v}, \Psi_{2 \mu-1,2 v}, \Psi_{2 \mu, 2 v-1}$ are symmetric in $\mu$, $v$; and

$$
\left\{\begin{array}{l}
\Psi_{2 \mu-1,2 v-1}+\Psi_{2 \mu, 2 v}=\frac{1}{2} \delta_{\mu \nu} \operatorname{Re}(d \Theta) \\
\Psi_{2 \mu-1,2 v}-\Psi_{2 \mu, 2 v-1}=-\frac{1}{2} \delta_{\mu \nu} \operatorname{Im}(d \Theta)
\end{array}\right.
$$

where $\left\{\Psi_{i j}\right\}$ is the second fundamental form in the canonical frame adapted to $(J, K)$ at $p$.

Proof. By definition, we have

$$
\begin{aligned}
\Psi_{2 \mu-1,2 v-1} & =\operatorname{Re}\left(\mathcal{C}_{\mu \nu}+\mathcal{D}_{\mu \nu}\right) \\
\Psi_{2 \mu, 2 \nu} & =\operatorname{Re}\left(-\mathcal{C}_{\mu \nu}+\mathcal{D}_{\mu \nu}\right) \\
\Psi_{2 \mu-1,2 v} & =\operatorname{Im}\left(-\mathcal{C}_{\mu \nu}-\mathcal{D}_{\mu \nu}\right) \\
\Psi_{2 \mu, 2 v-1} & =\operatorname{Im}\left(-\mathcal{C}_{\mu \nu}+\mathcal{D}_{\mu \nu}\right)
\end{aligned}
$$

Therefore, Equation (4.5) and (4.6) imply that all four 1-forms listed are symmetric in $\mu, \nu$. And the two equations follow immediately by adding and subtracting corresponding equations.

Corollary 4.4. Let $L^{2 n}$ be a hyper-Lagrangian in a hyperkähler manifold $M^{4 n}$ with defining map $J$. Let $K: L \rightarrow \mathbb{S}^{2}$ be any smooth map orthogonal to $J$ at every point of $L$. Then for any fixed point $p \in L$, the second fundamental form $\left\{h_{i j k}\right\}$ in the canonical frame adapted to $(J, K)$ at $p$ satisfies

$$
h_{i j k}=h_{j i k} \quad \text { for } \quad\{i, j\} \neq\{2 \mu-1,2 \mu\} \text { for any } \mu
$$

and

$$
h_{2 \mu-1,2 \mu, k}=h_{2 \mu, 2 \mu-1, k}-\operatorname{Im}\left(d \Theta\left(e_{k}\right)\right) .
$$

Moreover, for any $\mu, \nu$,

$$
h_{2 \mu-1,2 v-1, k}+h_{2 \mu, 2 v, k}=\delta_{\mu \nu} \operatorname{Re}\left(d \Theta\left(e_{k}\right)\right) .
$$


Proof. It is immediately from the symmetries of $\Psi$ that $h_{i j k}=h_{j i k}$ if $i+j$ is even. If $i+j$ is odd, then we consider $h_{2 \mu-1,2 v, k}-h_{2 v, 2 \mu-1, k}$. By the symmetries of $\Psi_{2 \mu-1,2 v}$ and equation for $\Psi_{2 \mu-1,2 v}-\Psi_{2 \mu, 2 v-1}$ we have

$$
h_{2 \mu-1,2 v, k}-h_{2 v, 2 \mu-1, k}=h_{2 \mu-1,2 v, k}-h_{2 \mu, 2 v-1, k}=-\frac{1}{2} \delta_{\mu \nu} \operatorname{Im}\left(d \Theta\left(e_{k}\right)\right) .
$$

So we conclude that $h_{2 \mu-1,2 v, k}=h_{2 \mu, 2 v-1, k}$ if $\mu \neq v$. Otherwise, we have

$$
h_{2 \mu-1,2 \mu, k}=h_{2 \mu, 2 \mu-1, k}-\operatorname{Im}\left(d \Theta\left(e_{k}\right)\right) .
$$

This completes the proof of the first part. The second part follows immediately from the corresponding equation for $\Psi$.

Note that the formula for commuting $i, j$ in $h_{i j k}$ in this corollary can also be obtained from Proposition 2.1.

It is interesting to compare the result in this corollary with the complete symmetry of all three indices of the second fundamental form of a special Lagrangian submanifold in a Calabi-Yau manifold. In our case, the second fundamental form of a hyper-Lagrangian does not have the complete symmetry in all three indices in general. However, the corollary shows that the second fundamental form has a lot of symmetry which is sufficient to implies the first-order equation on $\Theta$ relating $\partial \Theta$ to the mean curvature vector $H$ and the holomorphic symplectic form $\Omega_{J}$.

If we denote $d \Theta_{p}\left(e_{k}\right)=P_{k}-i Q_{k}$, then

$$
P_{k}=h_{11 k}+h_{22 k}=h_{33 k}+h_{44 k}=\cdots=h_{2 n-1,2 n-1, k}+h_{2 n, 2 n, k}
$$

and

$$
Q_{k}=h_{12 k}-h_{21 k}=h_{34 k}-h_{43 k}=\cdots=h_{2 n-1,2 n, k}-h_{2 n, 2 n-1, k}
$$

for all $k=1, \ldots, 2 n$. And we have

$$
d \Theta_{p}=\sum_{k=1}^{2 n}\left(P_{k}-i Q_{k}\right) \zeta_{k} .
$$

In terms of the forms $\phi_{v}$ and $\bar{\phi}_{v}$, this is given by

$$
\begin{aligned}
d \Theta_{p}= & \frac{1}{2} \sum_{v}\left[\left(P_{2 v-1}-Q_{2 v}\right)-i\left(P_{2 v}+Q_{2 v-1}\right)\right] \phi_{v} \\
& +\frac{1}{2} \sum_{v}\left[\left(P_{2 v-1}+Q_{2 v}\right)+i\left(P_{2 v}-Q_{2 v-1}\right)\right] \bar{\phi}_{v} .
\end{aligned}
$$

Hence, one concludes that

$$
\partial \Theta=\frac{1}{2} \sum_{v}\left[\left(P_{2 v-1}-Q_{2 v}\right)+i\left(P_{2 v}+Q_{2 v-1}\right)\right] \phi_{v} .
$$

Now we can prove the following.

Theorem 4.5. Let $L^{2 n}$ be a hyper-Lagrangian submanifold in a hyperkähler manifold $M^{4 n}$ and for any $p \in L, J(p)$ be the almost complex structure at $p$ such that $\left.\Omega_{J}(p)\right|_{T_{p} L}=0$. Then

$$
H\lrcorner \Omega_{J}+2 i \partial J=0,
$$


where $H$ is the mean curvature vector of $L$ in $M$.

Proof. We continue to work on the local representation $\Theta$ of the map $J: L \rightarrow \mathbb{S}^{2}$. The mean curvature vector is

$$
\begin{aligned}
H & =-\sum_{j=1}^{2 n}\left(\sum_{k=1}^{2 n} h_{j k k}\right) f_{j} \\
& =-\sum_{\mu=1}^{n} \sum_{k=1}^{2 n}\left[\left(h_{2 \mu-1, k k}-i h_{2 \mu, k k}\right) f_{\mu}^{\prime}+\left(h_{2 \mu-1, k k}+i h_{2 \mu, k k}\right) f_{\mu}^{\prime \prime}\right] .
\end{aligned}
$$

Therefore,

$$
\begin{aligned}
H\lrcorner \Omega_{J} & =i H\lrcorner \sum_{\mu=1}^{n} \phi_{\mu} \wedge \psi_{\mu} \\
& =-i \sum_{\mu=1}^{n} \sum_{k=1}^{2 n} \phi_{\mu}\left(h_{2 \mu-1, k k}-i h_{2 \mu, k k}\right) \\
& =-i \sum_{\mu=1}^{n} \sum_{\nu=1}^{n} \phi_{\mu}\left[\left(h_{2 \mu-1,2 v-1,2 v-1}+h_{2 \mu-1,2 v, 2 v}\right)-i\left(h_{2 \mu, 2 v-1,2 v-1}+h_{2 \mu, 2 v, 2 v}\right)\right] .
\end{aligned}
$$

If $\mu \neq \nu$, then by Corollary 4.4 and the symmetry of the last two index, one has

$$
h_{2 \mu-1,2 v-1,2 v-1}+h_{2 \mu-1,2 v, 2 v}=h_{2 \mu, 2 v-1,2 v-1}+h_{2 \mu, 2 v, 2 v}=0 .
$$

Hence, all the cross terms in the sum vanish and we have

$$
H\lrcorner \Omega_{J}=-i \sum_{\mu=1}^{n} \phi_{\mu}\left[\left(h_{2 \mu-1,2 \mu-1,2 \mu-1}+h_{2 \mu-1,2 \mu, 2 \mu}\right)-i\left(h_{2 \mu, 2 \mu-1,2 \mu-1}+h_{2 \mu, 2 \mu, 2 \mu}\right)\right] .
$$

Note that for any $\mu$,

$$
\begin{aligned}
P_{2 \mu-1}-Q_{2 \mu} & =\left(h_{2 \mu-1,2 \mu-1,2 \mu-1}+h_{2 \mu, 2 \mu, 2 \mu-1}\right)-\left(-h_{2 \mu-1,2 \mu, 2 \mu}+h_{2 \mu, 2 \mu-1,2 \mu}\right) \\
& =h_{2 \mu-1,2 \mu-1,2 \mu-1}+h_{2 \mu-1,2 \mu, 2 \mu}
\end{aligned}
$$

and

$$
\begin{aligned}
P_{2 \mu}+Q_{2 \mu-1} & =\left(h_{2 \mu-1,2 \mu-1,2 \mu}+h_{2 \mu, 2 \mu, 2 \mu}\right)+\left(h_{2 \mu, 2 \mu-1,2 \mu-1}-h_{2 \mu-1,2 \mu, 2 \mu-1}\right. \\
& =h_{2 \mu, 2 \mu-1,2 \mu-1}+h_{2 \mu, 2 \mu, 2 \mu} .
\end{aligned}
$$

Therefore, we have

$$
H\lrcorner \Omega_{J}+2 i \partial \Theta=0 .
$$

By the theorem, we immediately conclude the following.

Corollary 4.6. Let $L^{2 n}$ be a hyper-Lagrangian submanifold in a hyperkähler manifold $M^{4 n}$ and for any $p \in L, J(p)$ be the almost complex structure at $p$ such that $\left.\Omega_{J}(p)\right|_{T_{p} L}=0$. Then $L$ is minimal if and only if $J$ is anti-holomorphic. 
This corollary includes the case of complex Lagrangian submanifolds. In this case, $J$ is a constant.

In the case that $L$ is in fact a Lagrangian with respect to a fixed $\omega_{J_{o}}$. We may choose $J_{2}$ to be the fixed $J_{o}$. Then at the point $p \in L$, there is a function $\psi: L \rightarrow \mathbb{R}$ with $\psi(p)=\pi / 2$ such that $\Theta(x)=\sin \psi(x) J_{1}+\cos \psi(x) J_{3}$. It follows immediately that $d \Theta=-d \psi$ at $x=p$. These imply that $Q_{k}=0$ for all $k$ and $d \psi=-\sum_{k} P_{k} \zeta_{k}$. On the other hand, the holomorphic symplectic form at $p$ is given by $\Omega_{J}=\omega_{3}-i \omega_{2}$. So $\left.\left.\left.H\right\lrcorner \Omega_{J}=H\right\lrcorner \omega_{3}-i H\right\lrcorner \omega_{2}$ and by Theorem 4.5, we have

$$
\begin{aligned}
-H & \lrcorner \omega_{3}+i(H\lrcorner \omega_{2}\right) \\
& =2 i \partial \Theta \\
& =i \sum_{v}\left(P_{2 v-1}-i P_{2 v}\right) \phi_{v} \\
& =i \sum_{v}\left(P_{2 v-1}-i P_{2 v}\right)\left(\zeta_{2 v-1}+i \zeta_{2 v}\right) \\
& =\sum_{v}\left[\left(P_{2 v} \zeta_{2 v-1}-P_{2 v-1} \zeta_{2 v}\right)+i\left(P_{2 v-1} \zeta_{2 v-1}+P_{2 v} \zeta_{2 v}\right)\right] .
\end{aligned}
$$

Therefore

$$
H\lrcorner \omega_{2}+d \psi=0,
$$

which implies that $\psi$ is in fact the phase map up to a constant. Moreover, we have an additional equation

$$
H\lrcorner \omega_{3}=\sum_{v}\left(P_{2 v-1} \zeta_{2 v}-P_{2 v} \zeta_{2 v-1}\right) \text {. }
$$

\section{A remark on regularity}

Finally, we would like to make one remark about the regularity of the mean curvature flow of hyper-Lagrangian submanifolds under a natural condition on the associated map $J$. It was proved by M. T. Wang in [13] that a symplectic surface in a four-dimensional Kähler-Einstein manifold remains symplectic and does not develop any Type I singularity along the mean curvature flow. If the Kähler-Einstein is in fact hyperkähler, then a surface being symplectic is equivalent to the condition that the image of the map $J$ is contained in a hemisphere. To see this, we observe that any surface $L$ in a four-dimensional hyperkähler manifold is always hyper-Lagrangian. Therefore, there is a $J(x)=\sum_{\alpha=1}^{3} a_{\alpha}(x) J_{\alpha}$, with $a=\left(a_{1}, a_{2}, a_{3}\right) \in \mathbb{S}^{2}$ and an orthogonal set of parallel complex structures $\left\{J_{\alpha}\right\}_{\alpha=1}^{3}$ such that $J\left(T_{x} L\right)=T_{x} L$ for all $x \in L$. In a neighborhood of any point $x \in L$, we may choose an orthonormal frame for $T L$ of the form $\left\{e_{1}, e_{2}=J e_{1}\right\}$. Then

$$
\omega_{1,12}=\bar{g}\left(J_{1} e_{1}, e_{2}\right)=a_{1} \quad \forall x \in L .
$$

Therefore, $a_{1}>0$ if and only if $L$ is symplectic with respect to $\omega_{1}$. This shows the required equivalence. Therefore Wang's result can be rephrased as: If the image of the map $J$ on $L$ contained in a hemisphere, then the image $J$ on $L_{t}$ remains in the same hemisphere and does not develop any Type I singularity along the mean curvature flow. In the following, we will see that the proof in [13] actually can be applied to the higher dimensional hyper-Lagrangian submanifolds and gives the following.

Theorem 5.1. Let $\left(M^{4 n}, \bar{g}\right)$ be a hyperkähler manifold. Let $L_{t} \subset M^{4 n}, t \in[0, T)$ for some $T>0$, be a family of middle dimensional submanifolds given by the mean curvature flow. Suppose that $L_{0} \subset M$ is hyper-Lagrangian and the image of the corresponding map $J: L_{0} \rightarrow \mathbb{S}^{2}$ 
is contained in a hemisphere. Then the image of $J:[0, T) \times L_{0} \rightarrow \mathbb{S}^{2}$ is contained in the same hemisphere and the mean curvature flow does not develop any Type I singularity.

Proof. By Theorems 3.4 and 3.6, $L_{t}$ is hyper-Lagrangian for each $t \in[0, T)$ and $J(t, x)$ satisfies the harmonic map heat flow which can be written as

$$
\left(\partial_{t}-\triangle\right) a_{\alpha}=|\nabla a|^{2} a_{\alpha}, \quad \alpha=1,2,3,
$$

where $a_{\alpha}$ are components of $J$ as a map into $\mathbb{S}^{2} \subset \mathbb{R}^{3}$. If $J\left(L_{0}\right)$ is contained in a hemisphere which may be assumed to be $\left\{a \in \mathbb{S}^{2} \mid a_{1}>0\right\}$. Then we have $a_{1}(x)>0$ for all $x \in L_{0}$. Applying maximum principle to the harmonic map heat flow, we conclude that $a_{1}(t, x)>0$ for all $(t, x) \in[0, T) \times L_{0}$. This proves the first part of the theorem.

To see the second part, we observed that $L_{t}$ is hyper-Lagrangian for all $t$. Theorem 4.5 implies that

$$
H\lrcorner \Omega_{J}+2 i \partial \Theta=0 .
$$

Hence,

$$
|H|^{2}=2|\partial \Theta|^{2} \leq 2|\nabla a|^{2}
$$

This estimate implies

$$
\left(\partial_{t}-\triangle\right) a_{1} \geq \frac{1}{2}|H|^{2} a_{1} .
$$

Then standard argument using $a_{1}>0$ imply the nonexistence of Type I singularity, see, for example, [5], or the proof of Proposition 5.2 of [13].

The same argument is also applied to Lagrangian submanifold in a Calabi-Yau manifold $(M, \omega, \Omega)$ with $* \operatorname{Re} \Omega>0$. Firstly, it was proved by Smoczyk [11] that it remains Lagrangian along the mean curvature flow. Then Wang showed in [13], see also [14], that the condition $* \operatorname{Re} \Omega>0$ is preserved and the flow does not develop any Type I singularity using the same argument.

\section{Acknowledgments}

The authors would like to thank Mu-Tao Wang and André Neves for helpful discussions.

\section{References}

[1] Atiyah, M. Hyper-Kähler manifolds, in Complex Geometry and Analysis, (Pisa, 1988), 1-13, Lecture Notes in Math., 1422, Springer, Berlin, (1990).

[2] Chen, J. Deforming surfaces in four-dimensional manifolds, Symplectic and Contact Topology: Interactions and Perspectives, (Toronto, ON/Montreal, QC, 2001), 79-88, Fields Inst. Commun., 35, Amer. Math. Soc., Providence, RI, (2003).

[3] Chen, J. and Li, J. Mean curvature flow of surface in 4-manifolds, Adv. Math. 163(2), 287-309, (2001).

[4] Chen, J. and Tian, G. Moving symplectic curves in Kähler-Einstein surfaces, Acta Math. Sinica 16(4), 541-548, (2000).

[5] Ecker, K. and Huisken, G. Mean curvature evolution of entire graphs, Ann. of Math. (2) 130(3), 453-471, (1989).

[6] Hitchin, N. J., Karlhede, A., Lindström, U., and Roček, M. Hyper-Kähler metrics and supersymmetry, Comm. Math. Phys. 108(4), 535-589, (1987).

[7] Hitchin, N. J. Hyper-Kähler manifolds, Séminaire Bourbaki, Astérisque 206, 1991/92, Exp. No. 748, 3, 137-166, (1992).

[8] Hitchin, N. J. The moduli space of complex Lagrangian submanifolds, Sir Michael Atiyah: A great mathematician of the twentieth century, Asian J. Math. 3(1), 77-91, (1999). 
[9] Leung, N. C. Lagrangian submanifolds in hyperKähler manifolds, Legendre transformation, J. Differential Geom. 61(1), 107-145, (2002).

[10] Leung, N. C. Riemannian geometry over different normed division algebras, J. Differential Geom. 61(2), 289-333, (2002).

[11] Smoczyk, K. A canonical way to deform a Lagrangian submanifold, dg-ga/9605005.

[12] Thomas, R. P. and Yau, S.-T. Special Lagrangians, stable bundles and mean curvature flow, Comm. Anal. Geom. 10(5), 1075-1113, (2002).

[13] Wang, M. T. Mean curvature flow of surfaces in Einstein four-manifolds, J. Differential Geom. 57(2), 301-338, (2001).

[14] Wang, M. T. Mean curvature flow in high codimension, in Proceedings of ICCM2001, Stud. Adv. Math. International Press, 275-283, (2004).

\section{Received September 13, 2006}

Department of Mathematics and Institute of Mathematical Sciences,

The Chinese University of Hong Kong, Shatin, N. T., Hong Kong e-mail: leung@ims.cuhk.edu.hk

Department of Mathematics, The Chinese University of Hong Kong, Shatin, N. T., Hong Kong e-mail: tomwan@math.cuhk.edu.hk

Communicated by Peter $\mathrm{Li}$ 